\title{
Dictionnaire Guillevic
}

\author{
Bernard Fournier
}

Académie française : Grand Prix en 1975.

ACAdÉmie Mallarmé : refondée en 1975 par Jean Rousselot, Denys-Paul Bouloc, Michel Manoll, Marcel Béalu et Edmond Humeau. Guillevic en sera le premier Président, toujours prêt à démissionner, ce qu'il fera en 1993.

AlBERTINI, LUCIE : rencontrée en septembre 1969, traductrice du suédois, notamment d'Ingmar Bergman, seconde épouse en 1981. Très investie dans la carrière du poète, elle l'a aidé dans toutes ses activités (elle a notamment tenu scrupuleusement les minutes des réunions de l'Académie Mallarmé). Elle gère les archives du poète, publie ses inédits posthumes et fait vivre son œuvre en rassemblant autour d'elle tous les lecteurs de Guillevic.

AlÉMANIQUE : langue parlée dans le Sündgau, qui correspond aujourd'hui au département du Haut-Rhin. Guillevic l'a parlée durant ses années passées à Ferrette (1919-1926). Il le traduira littérairement plus tard, notamment Nathan Katz.

Alexandre Guillevic : frère du poète, né en 1910 à Jeumont, Nord, décédé à Colpo en 1959.

Allemand (traducteur) : notamment de Brecht aux éditions de l'Arche (Baal et Mère Courage), mais aussi d'Hölderlin, Goethe, Rilke. Sa pratique de la langue vient de son adolescence passée en Alsace et d'une année de service militaire comme interprète (à sa demande) à Mayence. 
Altкirch : chef-lieu d'arrondissement du Haut Rhin où le jeune Guillevic fit ses études, à partir de 1919, date à laquelle il entre en cinquième, jusqu'en 1925, quand il obtient un baccalauréat de Mathématiques élémentaires. Nombreux allers-retours Altkirch-Ferrette dans un tortillard qui lui permit de se familiariser avec la langue alémanique et un de ses poètes, Nathan Katz.

ANGERS : Jacques Lardoux organise le premier colloque international sur le sol français en 2002.

Anthologie de la poésie française de Simon Kra dans laquelle il découvre le vers libre en 1924.

Aragon, Louis (1897-1982) : le grand aîné tant sur le plan poétique que politique, mais dont il ne sent jamais très proche, à la différence d'Eluard. Préface les Trente et un sonnets en 1955. Guillevic a toujours contesté que ce soit sur l'ordre d'Aragon qu'il aurait écrit ces sonnets. Arland, Marcel (1899-1986) : ami de Guillevic, reconnu grâce à une photo des Nouvelles littéraires dans le train les emmenant, pendant la guerre, à l'école d'officier de Bouguenais (Loire Atlantique). Membre du Comité de Rédaction des éditions Gallimard, c'est grâce à lui que paraîtra Terraqué. Longue amitié jusqu'à sa mort en 1987.

Argile: premier titre de Terraqué, et annoncé comme tel dans une publicité d'Europe, en 1939.

ARMOIRE : c'est le premier mot du premier recueil, et qui lui vaut, à ce jour, sans doute le plus grand nombre de commentaires. À l'origine, ce texte n'était pas placé en tête.

Art poétique (1989) : recueil mais aussi thème de tout temps pratiqué (notamment, sous ce titre dans Terraqué, 1942 et Gagner, 1947).

ARTAUd, ANTONIn (1896-1948) : écrivain et acteur : Guillevic lui rend visite à l'hôpital de Rodez, où il est interné pour troubles psychiatriques, en 1945.

Auricoste, Marianne : comédienne, compagne du poète rencontré en 1965. Elle a composé des récitals à partir de textes de Guillevic, notamment à la Maison de la Poésie en 1999. A publié ses souvenirs dans Guillevic, Les Noces du goéland, L'Harmattan, 2007.

Autres (1980) : recueil qui rassemble quelques-uns des longs poèmes de Guillevic parmi les plus célèbres. Réédité avec Etier en poche dans la collection Poésie : Gallimard.

Avec (1966). 
BEAUCE : région découverte en 1953, où Guillevic habitera avec Jacqueline Woh dans une maison qu'il achète à la Forêt-Sainte-Croix. Il y accueillera de nombreux poètes et amis.

Bergeries, dans Autres, ensemble sur une forme unique et très remarqué. Billoux, FrançoIs : ministre communiste de la quatrième république qui appelle Guillevic à l'Economie Nationale, chargé de mission puis chef adjoint de cabinet chargé d'élaborer des lois sur les dommages de guerre et les problèmes d'urbanisme.

BoNAGUIL : 1970 premier hommage consacré à Guillevic.

Bouloc (Denys-Pol, 1918-2006), poète qui a beaucoup aidé au travail administratif de l'Académie Mallarmé.

Bourse Goncourt de la PoÉsie, en 1989.

Boxe : Guillevic fait de la boxe en 1922 pour se défendre contre ses coreligionnaires.

BRECHT, BERTOLt (1898-1956) : poète et dramaturge allemand (auteur de Mère courage et Baal), que Guillevic traduit à partir de 1954

BRETAGNE : malgré ses années alsaciennes puis parisiennes, Guillevic s'est toujours senti breton. Il n'en parlait pas la langue, mais a conservé des attaches sentimentales avec cette région d'une importance que Maria Lopo a soulignée dans son ouvrage, Guillevic et sa Bretagne. Il a toujours refusé cependant de se laisser enfermer dans le régionalisme se disant breton poète plutôt que poète breton.

BUCAREST : séjour en Roumanie en 1964

BudAPEST : séjours en Hongrie en 1963 et en 1970.

C

Cahiers de l'Ecole de Rochefort (1942) : publication d' "Ensemble » et de "Les Rocs» (préfacé par Jean Follain) qui viendront rejoindre Exécutoire en 1947. Jean Bouhier reprochera ensuite à Guillevic son aventure des sonnets. Mais Guillevic a toujours conservé d'excellentes relations avec chacun des membres de cette « école » : Michel Manoll, Jean Rousselot, par exemple.

Carnac : recueil de 1963 qui inaugure la période du retour aux sources bretonnes, après les années militantes. Après Terraqué, c'est son recueil le plus célèbre, mais qui tardera à s'imposer à la ville de Carnac même. Carnets : qu'il commence à remplir en 1923 (voir L'Expérience Guillevic, Deyrolles/Opales, 1994).

Cassou, Jean (1897-1986) : poète et écrivain, rencontré durant la guerre, il lui offre de publier dans Europe, Argile qui deviendra Terraqué. 
Célébrations Nationales : Guillevic est enregistré par les Archives de France pour le centenaire de sa naissance en 2007.

Centre National des Ecrivains : organisme fondé par la Résistance pour contrôler la politique d'édition. Guillevic en est membre en 1944, où il rencontre Aragon, Elsa Triolet et bien d'autres. Ne semble pas y avoir joué un rôle déterminant. Il en sera le trésorier jusqu'en 1970

Cerisy-LA-SAlle (Manche) : Centre Culturel International qui organise des colloques célèbres pendant l'été. Guillevic assiste à la décade sur " roman et poésie " en 1959, "Queneau " en 1960, "Art et psychanalyse " 1962. Un colloque avec Clancier et Tortel lui sera consacré. Un « jeu Guillevic » existe qui consiste à trouver le titre d'une œuvre littéraire à partir de mimes.

Cézanne, Paul (1839-1906) : peintre : Guillevic est très marqué par cette peinture, son travail et son esthétique.

Charleville-Mézières (chef-lieu des Ardennes) : ville de Rimbaud où Guillevic est nommé Receveur-Rédacteur de l'Administration de l'Enregistrement, en 1932. La ville lui rendra hommage en 1996, mais, déjà fatigué, il ne pourra s'y rendre.

Chant, Le (1990) : poursuite de la réflexion sur l'art poétique, mais cette fois, avec pour entrée, toujours le mot de « chant ».

Chaulot, Paul (1914-1969) : poète, inspecteur de police, ami de Guillevic.

Chevtchenko, Tarass : premier voyage en URSS en 1964 avec Frénaud et Caillois pour célébrer le poète ukrainien.

CinÉma : objet de plusieurs films dont le plus connu est celui de Serge Moati; voir aussi Pierre Rissient pour le film Cinq et la peau, 1977, dont Guillevic a écrit le scénario et pour lequel il a effectué un voyage en Extrême-Orient, à Manille plus particulièrement.

Claude-Bernard (rue, Paris $V^{\circ}$ ) : dernier domicile de Guillevic, où il emménage en 1980 avec Lucie Albertini.

Claudel, Paul (1868-1955) : poète et dramaturge. Guillevic lui envoie son premier texte, une "Ode à la vierge ", en 1922, dont on n'a pas encore retrouvé trace. Un des premiers maîtres de Guillevic.

Colomba Voronca : première maîtresse de Guillevic, épouse du poète Ilarie Voronca dont elle était séparée et sœur du poète Claude Sernet, rencontrée en 1938.

Colpo : village du Morbihan, à l'intérieur des terres, où habitaient certains membres de sa famille qu'il allait visiter avec ses parents. Son père en retraite s'y fait agent d'assurance. 
Communisme : adhésion au Parti clandestin pendant l'Occupation par l'intermédiaire d'André Adler. A lu Marx et croit jusqu'au bout à l'analyse marxiste de la vie économique.

Corfou (Italie), voyage à l'occasion du Huitième Congrès des Poètes. Creusement (1987) : un des deux recueils, avec Motifs que Guillevic donne à Gallimard pour son quatre-vingtième anniversaire. Ce mot théorise sa méthode poétique de concentration du vers et du verbe.

D

Dadelsen, Jean-Paul De (1913-1957) : poète rencontré très tôt à Altkirch, dans le train en 1924; les amis se perdront de vue jusqu’à la mort prématurée de l'auteur de Jonas.

Daix, Pierre : collègue et premier biographe de Guillevic dans Poètes d'Aujourd'hui, en 1954, mais qui prendra ses distances avec le militantisme. Son étude, jugée trop partisane, sera reprise par Jean Tortel dans la même collection, en 1971.

Drieu La Rochelle, Pierre (1893-1945) : romancier, reprend la Nouvelle Revue française à Jean Paulhan sous la volonté de l'Occupant, au moment où Guillevic fait sa connaissance. Se noue une étrange amitié entre ses deux hommes que tout séparait. Présente son adhésion au Parti Communiste au CNE.

Du domaine (1977) : recueil qui ouvre une autre période celle de l'âge et d'une certaine sagesse sous la forme nouvelle de "quanta".

\section{E}

Ecole (maternelle) de Carnac qui prend le nom de Guillevic en 1982. C'est la première d'une suite : il y aura notamment le collège de SaintJean-Brévelay en 1986, de Carnac et la médiathèque d'Hennebont.

ECONOMISTE, formation professionnelle acquise par des cours du soir, après l'Enregistrement et qui lui a permis de "monter " à Paris en 1935. C'est cette formation qui lui a donné des arguments pour son engagement politique. Détaché au Contrôle économique en 1942, il rédige des " précis " de procédure pénale et de droit pénal.

Eluard, Paul (1895-1952) : rencontré en 1941, grand ami de Guillevic qui le réconcilie avec le surréalisme. Il est un maître pour Guillevic.

EMinescu, Minai (1850-1889) : poète roumain : Guillevic assiste à son $75^{\circ}$ anniversaire à Bucarest.

Enregistrement du Domaine et du Timbre : reçu au concours de rédacteur en 1926, après une année sabbatique offerte par ses parents, 
Guillevic fera des intérims dans toute la région alsacienne : Huningue, Mulhouse,

Entretiens : genre de confessions que Guillevic apprécie pour la spontanéité et sa diversité. Il en a donné un grand nombre et à de nombreuses revues célèbres ou non.

ETATs-UNIS : pays capitaliste où il fait un premier séjour en 1968 dont il revient enchanté de New-York. Y retourne en 1975.

Etier (1979) : recueil qui fait référence aux canaux salins de Carnac.

Euclidiennes (1967) : recueil de formes mathématiques, unique en son genre.

Européen (Parlement) : Secrétaire Général de l'Association Mondiale des Poètes auprès du Parlement, 1991.

Exécutoire (1947) : diptyque qu'il forme dans la collection poésiel Gallimard, avec Terraqué; «Ils forment un tout » dit-il, pratiquement indissociable maintenant. C'est dans ce recueil qu'est paru un des textes les plus connus et de Guillevic sur la guerre : "Les Charniers ». Expérience, titre abandonné des premiers textes publiés en partie, tardivement repris en 1994 sous le titre L'Expérience Guillevic, par Jean-Louis Giovanonni et Pierre Vilar.

\section{F}

FABbri, JaCQUes : comédien qui met en scène un spectacle La Fête à Eugène, autour de ses textes, en 1990 au théâtre du Tourtour, Paris.

FERretTe : village du Sündgau où le père du poète fut nommé Maréchal des Logis chef en 1919; proche des frontières suisse et allemande, ce petit bourg se caractérise par deux lieux : l'ancien village avec sa motte féodale, et une refondation au XVII ${ }^{e}$ siècle dans la vallée. Guillevic a habité la gendarmerie puis une demeure dans le haut village. Après sa montée à Paris, il continuera d'y aller passer quelques jours de vacances.

Filles : deux filles issues de son union avec Alice Munch : Simone, née en 1932 et Irène en 1934.

Follain, Jean (1903-1971) : un des premiers poètes rencontrés à Paris grâce à René Massat au café Bonaparte. Il entretiendra une longue amitié avec le poète normand et lui consacrera deux articles et un ouvrage : Guillevic avec Jean Follain, Pierre-Alain Pingoud, CH-1009 Pully, 1993.

FrÉNAUd, ANDRÉ (1907-1993) : ami de Guillevic qui travaillait dans la même administration. Il eut une longue correspondance à propos des sonnets, par l'intermédiaire de la revue Europe.

\section{LittéRéalité}


FRONT POPULAIRE (1936) : sans se passionner pour la politique, il s'oriente vers un vote de gauche.

G

Gagner (1949) : recueil empreint de la période militante; l'édition revue et corrigée en 1981 s'en trouvera très largement diminuée.

Gallimard, Gaston (1881-1975) : éditeur qui a toujours pris tous ses livres. Sans doute avec quelques difficultés durant la période militante (pendant laquelle Seghers prendra le pas) mais jamais démentie.

GARA, LADISLAS : poète hongrois avec lequel il commence à traduire à partir d'une langue qu'il ne connaît pas.

Gaubert, Serge : professeur d'université à Lyon II, le premier à avoir consacré des journées d'étude sur l'ouvre de Guillevic qui lui dédicacera son recueil Le Chant en 1990. Il préfacera l'édition de poche poésie/Gallimard d'Art poétique, précédé de Paroi et suivi de Le Chant, en 2001.

GERMANISTE : pratiquant depuis son adolescence très rapidement tourné vers la traduction poétique ou dramatique.

GoETHE (Johann Wolfgang von, 1749-1832) : Guillevic traduit quelquesuns de ses poèmes.

Grorx (île de) : au large du Morbihan, Guillevic y fait de nombreux séjours de 1960 à 1967 : il y retrouve "sa " Bretagne.

GUERRES : en 1914, il a sept ans et habite à Saint-Jean Brévelay, son père est mobilisé restera à Chantilly. En septembre 1939, Guillevic est mobilisé à Dôle, fait l'école d'officier à Bouguenais, en Loire Atlantique, puis est nommé aspirant au Ministère de la Guerre à Paris. Guillevic est un des trois officiers à refuser la capitulation de Vichy. Se replie avec le Ministère à Terrasson en Dordogne pour revenir avec lui à Paris en juillet.

$\mathrm{H}$

Hachette (éditions) : rédige quelques travaux alimentaires pour cette maison, notamment sur les animaux, en 1970.

Heaney, Seamus (né en 1939) : poète irlandais, Prix Nobel de littérature en 1995, ami de Guillevic.

Heine (Heinrich, 1897-1856) : poète allemand que traduit Guillevic

HöLDERLIN (1770-1843), : grand poète romantique allemand que Guillevic traduit de façon permanente depuis 1954, parfois sur les mêmes poèmes (cf. Florilèges). 
Hongrie : invasion par les troupes de l'URSS en 1956 : Guillevic est d'accord car il croit au complot.

Hongrois (mes poètes) (éd. Corvina, Budapest, 1967) : recueil de choix de poèmes dans une nouvelle technique de traduction, à deux avec un bilingue et surtout un poète.

Hôpital, (éd. Arfuyen, ill. de Baltazar, 1988) : recueil qui relate la vue sur le Val de Grâce depuis son appartement de la rue Claude-Bernard. Un séjour au Val-de-Grâce, pendant son service militaire lui permit de lire des revues surréalistes; un autre à La Salpétrière en 1944 pour une crise d'appendicite; à la fin de sa vie, des séjours trop répétés à partir du début 1997, à La Salpétrière.

Humanité NOUVElle : mouvement communiste chrétien qui attire Guillevic en 1936, mais qui s'en détache après sa condamnation par le pape.

HUMOUR BLANC : sorte d'humour contre l'humour noir d'André Breton, et matérialisé par Jacques Lardoux dans ses entretiens : Humour terraqué Presses Universitaires de Vincennes-Saint-Denis, 1997.

L'Honneur des poètes: publication clandestine fondée par Paul Eluard qui y insère des poèmes de Guillevic sous le pseudonyme de Serpières.

I

Inclus (1973) : recueil qui poursuit la période des quanta et l'inspiration métaphysique.

INNOCENT : thème de la culpabilité et de la nostalgie d'un paradis de l'enfance perdue, partie de Possibles futurs, 1996, parue d'abord en 1993 (éd. Deyrolles, ill. de Marc Grandchamps).

INSPECTEUR DES IMPÔTS : fonction exercée depuis 1946 qui lui a permis de faire beaucoup de voyages, notamment dans la France d'Outre-Mer de l'époque (Maghreb aujourd'hui) pour la construction. Travaillait rue de Rivoli, dans ce qui est maintenant le Musée du Louvre. Lieu qui lui permit d'aller voir les ouvres d'art pendant ses heures de repas. Détaché dans les ministères communistes, il reviendra à l'Inspection de l'Economie nationale en 1947.

Irène (1935-1967) : deuxième fille de Guillevic, décédée le 30 mars 1967 (32 ans).

J

JEUMONT : commune du Nord, à la frontière belge, première affectation du gendarme Guillevic en 1910. Climat assez difficile à cause des grèves. 
$\mathrm{K}$

Katz, Nathan : poète de langue alémanique dont Guillevic fait la connaissance en 1922, dans le train de Ferrette à Altkirch. Il le traduit sérieusement à partir de 1954

Korrigans (Danse des) : un des rares livres consacré aux légendes bretonnes, directement, et destiné aux enfants.

$\mathrm{L}$

LA Fontaine (1621-1695) : poète imité dès 1915, repris en exergue de Vivre en poésie et à qui est dédicacé Art poétique.

LAMARTINe (Alphonse de, 1790-1869) : premier engouement pour un poète, 1922.

LANGUE : grand intérêt pour toutes les langues : " et toute langue est étrangère " pour celui qui entendait ses parents parler breton qu'on lui interdisait d'apprendre, et qui fut confronté à l'allemand et à l'alémanique!

LÉGER, Fernand (1881-1965) : peintre ami, avec qui il a réalisé Coordonnées, éd. Des trois Collines, Le Point d'or, Genève-Paris, 1947.

Lenau (Nikolaus Niembsch von Strehlenau, dit Nikolaus, 1802-1850) : poète autrichien que Guillevic traduit.

Lexiquer: (éd. La tuilerie topicale, Saint-Martin de Castillan, ill. de JeanMairs Le fage, 1986) petit livre sur l'alphabet qui prouve l'intérêt pour la langue.

Livet, Jean-Philippe : accueille dans Le Pont Mirabeau des poèmes de Guillevic, donnés par Follain.

M

"MaGnificat ": suite de poèmes parue isolément (éd. Carmen-Martinez, ill. Le Yaouanc, 1976, repris in Trouées), aux accents métaphysiques

MaI 1968 : les événements ont beaucoup marqué le poète. Juste à la retraite, Guillevic va pouvoir agir réellement : refondation de la SGDL, création de l'Union des écrivains, prend d'assaut l'Hôtel de Massa.

Maintenant (1993) : recueil important qui fait part large à la guerre de l'exYougoslavie.

MAISON DE LA POÉSIE : initiée par Guillevic et inauguré avec sa présidence, d'abord dans les Halles rénovées, puis au théâtre Molière. Hommage lui sera rendu en 1996 avec Lionel Ray et Jean-Michel Maulpoix.

MALGRÉ : projet de recueil qui comblerait le trou de la période dite des « basses eaux », à la fin des années cinquante. 
Mallarmé (Stéphane, 1842-1898) : Guillevic refondera l'Académie Mallarmé en 1975. Il fera rachater la maison de Valvins consacrée à un musée, maintenant propriété du département de Seine-et-Marne.

MARIE-Clotilde : premier amour de Guillevic (1921) qui mourra jeune (en 1924). Elle influencera nombre de ses écrits, associés aux violettes, par exemple «Elégies » dans Sphère et « Déjà » dans Trouées.

Marrakech (Maroc) : voyage en 1984 pour le Septième Congrès Mondial des Poètes.

Marx (Karl 1818-1883), marxisme : lit Le Capital dans les années du Front populaire, d'abord dans les Morceaux choisis par Henri Lefebvre. Il s'est toujours dit marxiste même après son départ du Parti Communiste en 1990.

Menton : lauréat du Collège poétique, y prononce un discours : « la poésie et le social », 1965.

Mère (Jeanne David, Cloucarnac, 1882-Colpo, 1942) : semble avoir des rapports ambigus avec sa mère entre haine et amour filial, dont les diatribes de Vivre en poésie sont le signe.

Messidor, : organe du Livre Club Diderot, dont il sera le directeur en 1970.

Militant : depuis la Résistance, Guillevic s'inscrit au Parti Communiste jusqu'après l'invasion de l'Afghanistan en 1979.

Milosz : (Oscar Vladislas de Lubicz-Milosz 1877-1939), un des maîtres de Guillevic.

Mont Saint-Michel : Prix en 1962, qui lui permet un séjour durant lequel il écrira Sphère.

MontréAl : 1967, colloque sur les " poètes et la terre ", rencontre mondiale de poésie à l'occasion de l'exposition universelle.

Mort : le 19 mars 1997. Eugène Guillevic meurt à 8 h 30, d'un arrêt cardiaque. Peu de jours auparavant il avait écrit : «Le temps s'étrangle/ Le temps s'arrête/ Mourons. " et "J'ai l'angoisse existentielle et terre ". La nouvelle ne sera diffusée que le lendemain dans les radios et les journaux. Beaucoup de monde va voir sa dépouille exposée à son domicile. 25 mars : cérémonie d'incinération au cimetière du PèreLachaise. La grande salle est comble : lectures de Raymond Jean, Yvon Le Men, Sylvie Girouard, la petite-fille du poète, Marianne Auricoste, André Libérati, Monique et Jean Royer, Jacques Lardoux, JeanFrançois Minot, Georges-Emmanuel Clancier. Les cendres seront déposées dans la lande de Carnac en novembre 2006.

Motifs (1987) : un des deux recueils avec Creusement que Guillevic donne à Gallimard pour son quatre-vingtième anniversaire. Il donne la parole 
à différents éléments naturels, tels la mer, la montagne, le lac, l'oiseau, etc.

Munch, Alice (1898-1981) : première épouse de Guillevic, rencontrée en 1924, mariage en 1930, décède en 1981. Elle était alsacienne et a élevé ses deux filles à Paris.

\section{$\mathbf{P}$}

Paroi (1970) : recueil métaphysique, en lien avec Inclus (1973).

Parti Communiste Français : adhère en 1942 dans la clandestinité, fonde la cellule du PCF au Ministère de l'Economie Nationale, et le quitte en 1979 après l'invasion des troupes soviétiques en Afghanistan. Paulhan, Jean (1884-1968) : directeur de La Nouvelle Revue française qui lui refusera d'abord ses textes, avant de le connaître en 1941 par l'intermédiaire de Drieu La Rochelle, qui avait pris les commandes de la revue placée par l'Occupant.

Peintres : relation soutenue depuis dès le début avec la découverte de Cézanne et Van Gogh, puis le groupe d'Altkirch, et enfin Picasso, Léger, Braque, Bazaine, etc. Nombreux livres illustrés, inaugurés par Coordonnées par Fernand Léger. Une exposition sur le travail accompli avec les peintres sera organisée à Villeneuve-lès-Avignon en 1988. Le recueil Relier, 2007, reprend une partie de l'abondante collaboration avec les peintres.

Père : (Eugène Guillevic), (1884-1958), d'abord marin puis gendarme et enfin agent d'assurance.

Picasso, Pablo (1881-1973) : devait réaliser une œuvre avec Guillevic, Les Charniers, en 1945, au vu du retour des déportés à Paris, projet qui n’a pas abouti.

PoÈte OfFiciel (1981) : peut-on dire que l'élection de Mitterrand a fait de Guillevic un poète officiel, avec son voyage en Hongrie avec le président Mitterrand en 1982.

Politique : militant durant quarante ans, Guillevic a toujours mis de la politique dans ses entretiens.

Ponge, Francis (1899-1988) : publie Le Parti pris des choses la même année que Terraqué; mais les deux poésies sont radicalement différentes.

Possibles futurs (1996) : dernier recueil anthume.

Présent (2004) : recueil posthume.

PRIX : (Grand) National de Poésie, institué à son instigation auprès de Jack Lang, ministre de la culture de François Mitterrand, en 1981. Il en sera 
récipiendaire en 1984. (voir aussi Bourse Goncourt), Prix Atlantida à Ténérife, 1992. Prix Supervielle à Oloron Sainte-Marie, 1992.

Prose : a priori, Guillevic n'a jamais écrit de prose. Mais les archives nous montrent qu'il s'est essayé au poème en prose Proses ou Boire dans le secret des grottes, posthume, Fishbacher 2001, et qu'il a rédigé nombre d'articles critiques ou des préfaces.

Publication : Premier ouvrage, une plaquette chez Tschann aux Cahiers de Sagesse en 1938 Requiem.

Q

Quatre-VInGts ans : en 1987, fêté par deux publications : Motifs et Creusement, et à Carnac, par une réception à la mairie et une lecture dans les menhirs.

Quotidiennes (2002) : premier recueil posthume, sur le même thème que Présent.

$\mathrm{R}$

Receveur de l'Enregistrement : premier emploi de Guillevic à Rocroi, dans les Ardennes, en 1930. Nommé Receveur-Rédacteur à Mézières en 1932.

RÉDACTEUR PRINCIPAL : c'est le concours qui lui permet de monter à Paris en 1935, nommé à la Direction Générale de l'enregistrement au Ministère des Finances. Secrétaire de la Commission des valeurs mobilières étrangères, qui fixe les charges fiscales de ces sociétés. Il est ainsi amené à côtoyer tout le fonctionnement du monde du capital. Il rédige des mémoires pour la Cour de Cassation, et des « codes».

Refus : sait-on que ses premiers textes ont été refusés par Jean Paulhan en 1932? puis par Les Cahiers du Sud et même par Seghers encore jusqu'en 1939?

ReLigion : selon les coutumes de l'époque, fortement ancrées en Alsace et en Bretagne, Guillevic pratique la religion catholique jusqu'à son engagement politique en 1936, peut-être même après. Il ne les reprendra plus mais avec son grand âge, il reviendra à son vocabulaire : "Magnificat », royaume, "Vitrail ».

Rennes : l'Université bretonne célèbre le centenaire de Guillevic par un colloque sur deux lieux, le campus et Carnac.

Requiem : première plaquette publiée en 1939 chez Tschann aux Cahiers de Sagesse, écrite en 1938 à Colpo, alors qu'il disait dans ses Carnets qu'il n'écrirait jamais de plaquette. Bien que republiée sur le tard, dans 
Lire Guillevic sous la direction de Serge Gaubert en 1983, repris dans Relier (2007).

Requis (1983) : un recueil important de Guillevic sur son questionnement métaphysique et matérialiste.

RésistanCE : se plaint d'en faire trop peu. Il aurait transporté des documents, fait de faux papiers.

RETRAITE : à partir de 1963, il bénéficie d'un congé spécial de quatre ans avant d'attendre 1967 ses soixante ans

Reverdy, Pierre (1889-1960) : réfugié à Solesmes, Guillevic le rencontre en 1944.

Revues : commence ses abonnements aux Nouvelles littéraires et à $\mathrm{La}$ Nouvelle revue française dès 1926, publie ses premiers textes dans $L a$ Vache bleue (non encore répertoriée) et Les Pyrénées littéraires (1927 pour « L'Hymne du hibou à la nuit » d'après un exercice scolaire), puis dans La Grive (Charleville, 1933) Le Pont Mirabeau (1934, pour les premiers textes qui seront repris dans Terraqué) et dans Commune (1939). S'intéressera toujours beaucoup au monde des revuistes qu'il soutiendra de ses textes.

Rumbaud (Arthur, 1854-1891) : le grand : celui qui l'a dégagé de la poésie classique apprise à l'école, celui qui l'a aidé à dépasser le surréalisme avant de l'avoir connu. A participé à de nombreuses manifestations autour de son ouvre, notamment lors du centenaire de sa mort pour une plaque sur la maison qu'il habita à Aden, et sur laquelle il demande que soit apposée une plaque. A habité quelques années lui-même à Charleville-Mézières (1932-1935).

Rissient, Pierre : cinéaste pour lequel il écrit le scénario de Cinq et la peau, 1977, à propos duquel il fait un séjour en Malaisie.

Rutsos, Yannis (1909-1990) : poète grec dont il fête le 70 e anniversaire à Athènes en 1979. Il y parle en représentant le Parti Communiste Français.

ROUMANIE : séjour en 1968.

$\mathrm{S}$

SAGESSE : groupe de poètes qui s'essayaient à former une école après (et contre) le surréalisme (comme "La nouvelle origine " avec Audiberti ou "l'Ecole de Rochefort "): René Massat, René Méjean, Maurice Fombeure. Guillevic ne s'y agrège pas tout à fait, aimant davantage les contacts que les écoles. Mais il y lit ses textes qui sont remarqués avant leur publication. 
Saint-Jean Brévelay : à l'intérieur des terres, dans le Morbihan, village breton où Guillevic a passé une partie de son enfance (entre 19121919). Il y avait de la famille. Deuxième affectation du gendarme Guillevic en 1912.

SENGHor, LÉOpold SÉDAR (1906-2001) : poète sénégalais, membre de l'Académie française et de l'Académie Mallarmé. C'est à ce dernier titre qu'il invite toute l'Académie à un voyage au Sénégal en 1980. Guillevic lui dédiera Qui, en 1988.

Service militaire dans les COA (Commis et Ouvriers d'Administration) à Besançon en 1927.

Simone : première fille du poète née en 1932.

Somlyo : poète hongrois né en 1920, ami de Guillevic.

SonNETs : pratique assidue durant les années militantes, certains ont dit sous l'influence d'Aragon, ce que Guillevic a toujours démenti : c'est un exercice qui lui permet de faire des gammes; Bertrand Degott a retrouvé un bon nombre des " cent sonnets » et plus que Guillevic écrivait le matin pour se mettre en forme!

SPHÈre (1963) : célèbre avec "Chemins " la fin de la période militante pour ouvrir celle des retrouvailles avec la Bretagne. Cette image du cercle, et par conséquent du centre, sera prééminente chez Guillevic.

STADLER (Ernst) : poète que traduit Guillevic.

StaLIne (Jossif Vissarionovitch Dougachvli, 1879-1953) : Guillevic écrira une ode au "Camarade Staline " pour ses 70 ans dans Les Lettres Françaises: il la reniera. La dénonciation de ses crimes au XX Congrès du PC de l'URSS lui fera perdre son enthousiasme mais pas ses convictions.

STRASBOURG : convalescence en 1928 après des problèmes d'intestins : il y découvre les opéras de Wagner et toute la mythologie celtique.

STRUGA : en Macédoine yougoslave en 1967. Y retourne en 1968 pour les rencontres poétiques de Struga sur le lac d'Ohrid. Couronne d'Or du Festival Internationale en 1976.

Sullivan (Jean) : poète prêtre, ami de Guillevic qui avait initié les entretiens de Choses Parlées.

Supervielle, Jules (1884-1960) : son maître en 1937. Il le rencontre grâce à René Jourdain et Pierre Albert-Birot; il lui dédiera Requiem, sa première plaquette. Il recevra le Prix Supervielle, à Oloron SainteMarie en 1992.

SURRÉALISME : rejeté d'emblée par la lecture de Rimbaud. Mais amitié avec Eluard et admiration pour Aragon. Peu de rapport avec Breton.

Sylvie, : petite fille du poète, née le 22 février 1961. 
Tchécoslovaquie (invasion en 1968) : Guillevic cette fois exprime son désaccord. S'y déplace avec l'Union des Ecrivains en 1969.

Terraqué, premier recueil, 1942, publié sous l'Occupation grâce à Drieu La Rochelle et Marcel Arland. Il devait paraitre en 1939 avec une prépublication en revue dans Europe. Ce furent «Les Charniers » qui prirent cette place en 1947. Recueil le plus célèbre de Guillevic dont il estime qu'il est la base de toute son ouvre à venir, et quili aura mis vingt ans à construire.

Tillon, Charles, (1897-1993) : ministre communiste de la Reconstruction qui appelle Guillevic à son service.

ToronTo : voyage en 1975 après New-York. Lieu du premier colloque post mortem, organisé par Sergio Villani, de l'Université York.

Tortel, Jean, (1904-1993) : poète et ami depuis 1938. Il ira souvent passer quelques jours de vacances chez lui à Villeneuve-lès-Avignon, notamment en 1955. Fête ses 80 ans à Avignon.

TrakL (Georg, 1887-1914) : poète autrichien que Guillevic a traduit.

Traduction : commence par la langue allemande, dès 1932, avec Trakl, Goehte, Lenau et Hölderlin. Il établira ensuite une méthode selon laquelle le poète a le dernier mot sur le traducteur littéral, ce qui lui permet de traduire dans des langues qu'il ne connaît pas, par exemple des poètes hongrois ou serbo-croates.

Trouées (1981) : qui inclut les deux célèbres ensemble "Vitrail " et " magnificat".

\section{$\mathrm{U}$}

UNESCO : hommage par Adonis et Marmoud Darwich, en 1990.

UnGARETTI (Giuseppe, poète italien, 1888-1970) : ami de Guillevic qui aura une certaine influence sur la réduction de son vers.

UNION DES ÉCRIVAINS : membre fondateur en mai 1968.

URSS : 3 voyages en 1964, 1966, 1972.

V

Valdalhon (camp) (Doubs): Guillevic y effectue sa période militaire 1939.

VaLÉRY, PAul (1871-1945) : poète, que Guillevic a connu à la fin de sa vie et qui l'a encouragé. Le poète vieillissant mais qui n'acceptait pas l'Occupant, a aidé Guillevic à faire libérer André Adler. Guillevic sera invité comme Président d'honneur du colloque sur le Centenaire de Valéry, à Montpelllier en 1994. 
VAN Gogh (1853-1890) : découverte déterminante de la peinture moderne en 1927 à Bâle.

Vitrail : dans la lignée de "Magnificat ", reprise des mots de la religion pour exprimer des accents métaphysiques, inclus dans Trouées (1981).

VIlLE, (1969) : poème écrit pour pouvoir vivre à Paris, malgré ses difficultés.

Vivre en poésie (1980) : premier livre d'entretiens publiés, avec Alain Vircondelet et Lucie Albertini. Grande influence sur les lecteurs potentiels. Premiers aveux d'un poète, jusque-là secret sur sa vie privée.

Voronca, Colomba : docteur ès sciences à l'Institut Pasteur, sœur du poète Claude Sernet et épouse d'un autre poète d'origine roumaine, Ilarie Voronca, compagne de Guillevic à partir de 1947; il vit avec elle à Vincennes.

WoH, JACQUeLINe (-2004) : compagne de Guillevic avec qui il vit à partir de 1952 et avec qui il partage la maison de la Forêt Sainte-Croix, dans la Beauce.

\section{Y}

YLIÈs, GulYa : poète hongrois qui l'invite sur le lac Balaton, en Hongrie, dans une maison d'écrivains.

YougosLAVIE : nombreux séjours aux rencontres internationales de Struga en Macédoine, amitié avec les poètes du lac d'Ohrid. La guerre l'a beaucoup marqué, ce qui s'entendra dans Maintenant.

Yrissou, Henri : directeur du service de la coordination des affaires économiques de la métropole et des pays d'Afrique du Nord, la Tunisie et le Maroc, dans les négociations avec l'étranger. Ce travail se poursuit de 1949 à 1963, date de l'Indépendance de l’Algérie. 